\title{
Phenotype-specific adverse effects of XPD mutations on human prenatal development implicate impairment of TFIIH-mediated functions in placenta
}

\begin{abstract}
Roxana Moslehi ${ }^{\star 1,2}$, Anil Kumar ${ }^{1}$, James L Mills ${ }^{3}$, Xavier Ambroggio ${ }^{4}$, Caroline Signore ${ }^{5}$ and Amiran Dzutsev ${ }^{6}$
Mutations in XPD (ERCC2), XPB (ERCC3), and TTD-A (GTF2H5), genes involved in nucleotide excision repair and transcription, can cause several disorders including trichothiodystrophy (TTD) and xeroderma pigmentosum (XP). In this study, we tested the hypothesis that mutations in the XPD gene affect placental development in a phenotype-specific manner. To test our hypothesis and decipher potential biologic mechanisms, we compared all XPD-associated TTD $(n=43)$ and XP $(n=37)$ cases reported in the literature with respect to frequencies of gestational complications. Our genetic epidemiologic investigations of TTD and XP revealed that the exact genetic abnormality was relevant to the mechanism leading to gestational complications such as preeclampsia. Through structural mapping, we localized the preeclampsia-associated mutations to a C-terminal motif and the helicase surfaces of XPD, most likely affecting XPD's binding to cdk-activating kinase (CAK) and p44 subunits of transcription factor (TF) IIH. Our results suggested a link between TTD- but not XP-associated XPD mutations, placental maldevelopment and risk of pregnancy complications, possibly due to impairment of TFIIH-mediated functions in placenta. Our findings highlight the importance of the fetal genotype in development of gestational complications, such as preeclampsia. Therefore, future studies of genetic associations of preeclampsia and other placental vascular complications may benefit from focusing on genetic variants within the fetal DNA.
\end{abstract}

European Journal of Human Genetics (2012) 20, 626-631; doi:10.1038/ejhg.2011.249; published online 11 January 2012

Keywords: trichothiodystrophy; xeroderma pigmentosum; XPD; TFIIH; human fetal development

\section{INTRODUCTION}

Mutations in XPD (ERCC2), XPB (ERCC3), and TTD-A (GTF2H5), genes involved in the nucleotide excision repair (NER) pathway and in transcription, are associated with trichothiodystrophy (TTD), a rare (affected frequency of 1 in $10^{6}$ ) disorder characterized by hair, skin and neurological abnormalities. ${ }^{1}$ Besides TTD, mutations in XPD (ERCC2) can lead to several other DNA repair disorders including xeroderma pigmentosum (XP), Cockayne syndrome (CS), cerebroocular facial syndrome (COFS) or a combination of XP/TTD, XP/CS, or COFS/TTD. ${ }^{1-4} X P D, X P B$, and TTD- $A$ code for components of the seven-subunit core complex of transcription factor (TF) IIH. TFIIH also contains a three-subunit Cdk-activating kinase (CAK) complex composed of cdk7, MAT1, and cyclin H. ${ }^{5}$ TFIIH has a role in NER and in both basal and activated transcription; ${ }^{6}$ as a component of RNA polymerase-II (Pol-II) transcription machinery, TFIIH has an important role in RNA Pol-II elongation. ${ }^{7}$ In addition, TFIIH is an important regulator of the cell cycle. ${ }^{5}$ The core complex is sufficient to support the NER function of TFIIH, while the CAK complex is required for phosphorylation of RNA Pol-II and numerous nuclear receptors (NRs). ${ }^{7}$
We recently reported ${ }^{8}$ that pregnancies in which the fetus had TTD were at significantly increased risk of preeclampsia, HELLP syndrome (a severe form of preeclampsia with hemolysis, elevated liver enzymes, and low platelets), and elevated mid-trimester maternal serum human chorionic gonadotropin levels. The affected fetuses had decreased fetal movement and were significantly more likely to be small for gestational age (SGA) and to deliver pre-term. ${ }^{8}$ Gestational abnormalities were only detected in TTD-affected pregnancies (where infant had two mutations) but not in unaffected pregnancies (where the infant was either a heterozygote or noncarrier), ${ }^{8}$ highlighting the relevance of the fetal genotype to development of observed complications.

Impairment of placental development could explain the constellation of abnormalities observed in TTD-affected pregnancies; ${ }^{8}$ thus, we hypothesize that mutations in TTD NER/transcription genes affect placental development. Given that pregnancies resulting in XP/TTD, also caused by mutations in $X P D$, were not associated with gestational complications, ${ }^{8}$ we further propose that both the fetal genotype and the exact genetic abnormality are relevant to the underlying mechanism.

\footnotetext{
${ }^{1}$ Department of Epidemiology and Biostatistics, School of Public Health, University at Albany, Albany, NY, USA; ${ }^{2}$ Cancer Research Center, University at Albany, Albany, NY, USA; ${ }^{3}$ Epidemiology Branch, Division of Epidemiology, Statistics and Prevention Research, Eunice Kennedy Shriver National Institute of Child Health and Human Development (NICHD), NIH, DHHS, Bethesda, MD, USA; ${ }^{4}$ Bioinformatics and Computational Biosciences Branch (BCBB), National Institute of Allergy and Infectious Disease (NIAID), NIH, DHHS, Bethesda, MD, USA; ${ }^{5}$ Pregnancy and Perinatology Branch, Center for Developmental Biology and Perinatal Medicine, Eunice Kennedy Shriver National Institute of Child Health and Human Development (NICHD), NIH, DHHS, Bethesda, MD, USA; ${ }^{6}$ Science Application International Corporations (SAIC), Cancer Inflammation Program, National Cancer Institute (NCI), NIH, Frederick, MD, USA

*Correspondence: Dr R Moslehi, Department of Epidemiology and Biostatistics, School of Public Health, and Cancer Research Center, University at Albany, Albany, NY 12144, USA. Tel: +1 518591 7225; Fax: +1 518591 7201; E-mail: rmoslehi@albany.edu

Received 31 August 2011; revised 27 October 2011; accepted 25 November 2011; published online 11 January 2012
} 
To test our hypotheses, we compared all XPD-associated TTD and XP cases reported in the literature with respect to frequencies of gestational complications. We then conducted an analysis of the correlation between XPD mutations and the presence of preeclampsia in all the reported cases in order to create the first structural map of preeclampsia-associated domains on the three-dimensional (3D) protein structure of XPD.

\section{MATERIALS AND METHODS}

\section{Comparison of gestational complications in $X P D$-associated} TTD versus XPD-associated XP

Study population. The study population consisted of all TTD, COFS/TTD, $\mathrm{XP}, \mathrm{XP} / \mathrm{CS}$, and $\mathrm{XP} / \mathrm{TTD}$ cases with mutations in XPD identified through a systematic literature review using Pubmed on MEDLINE. All case reports in English and French were included; reports in other languages were included if the abstract was in either English or French. A total of 43 TTD, ${ }^{9-22} 37 \mathrm{XP}^{23-35}$ six XP/TTD, ${ }^{3,8}$ four XP/CS, ${ }^{36-38}$ and $1 \mathrm{COFS} / \mathrm{TTD}^{8}$ patient with defects in the $X P D$ gene were identified and included in our study population.

Data collection. Data were obtained by reviewing the case reports using a standard abstraction form, collecting information on reproductive, pregnancy, and delivery histories of each affected pregnancy. Variables for analyses, created from abstraction forms, included presence of preeclampsia, HELLP syndrome, pre-term delivery $(<37$ weeks gestation), low birth weight $(<2500 \mathrm{~g})$, SGA $<10$ th percentile, SGA $<3$ rd percentile, decreased fetal movement, abnormal maternal serum triple screen test results, and history of neonatal intensive care unit admission. The variables were analyzed as present or absent from the record, or do not know if the report did not specifically mention the variable.

Statistical analysis. Frequencies of gestational complications were compared between XPD-associated TTD $(n=43)$ and XP $(n=37)$ cases. Analysis was done two ways: first by combining all 'do not know' and 'no' responses together for comparison with 'yes', and second by excluding all 'do not know' responses from the analyses. Statistical Analysis System (SAS) software (version 9.1, SAS Institute, Cary, NC, USA) was used for statistical comparisons; all $P$-values were calculated using Fisher's exact test and all tests were two sided.

\section{Mapping of preeclampsia-associated domains on XPD}

In order to limit variability caused by heterogeneity of observed pregnancy complications, structural mapping was restricted to preeclampsia, which was the most common well-documented prenatal complication among our subjects. All XP and TTD cases with specified mutations in the XPD gene and information on preeclampsia were included in the analysis. T-Coffee, ${ }^{39}$ Promals3D, ${ }^{40}$ NCBI BLAST, and TM-Align ${ }^{41}$ were used for generating structure-based multiple sequence alignments and mapping of the mutations in the $X P D$ gene to the available XPD crystal structures (PDB IDs: $3 \mathrm{CRV}^{42}{ }^{2} \mathrm{VSF}^{43}$ and $\left.2 \mathrm{VL}^{44}\right)$. PyMOL and Jalview ${ }^{45}$ were used for depiction of mapped XPD mutations and sequence alignments, respectively.

\section{RESULTS}

Comparison of gestational complications in $X P D$-associated TTD versus XPD-associated XP

Comparison of the frequencies of gestational complications between 43 TTD and $37 \mathrm{XP}$ cases with mutations in XPD revealed a significantly higher incidence of preeclampsia $(P=0.003)$, abnormal maternal serum triple screen test results $(P=0.02)$, pre-term delivery $(P<0.0001)$, low birth weight $(P<0.0001)$, SGA $(<10$ th percentile $)$ $(P=0.006)$, SGA $<3$ rd percentile $(P=0.003)$, and NICU admission $(P<0.0001)$ among $X P D$-associated TTD pregnancies (Table 1$)$. It is noteworthy that all reported gestational complications were found exclusively among TTD cases (Table 1).

Excluding cases without definitive information on the presence or absence of each complication produced similar results, although
Table 1 Comparison of pregnancy complications between TTD and $\mathrm{XP}$ cases with mutations in XPD

\begin{tabular}{|c|c|c|c|}
\hline & $T T D(\mathrm{n}=43)$ & $X P(\mathrm{n}=37)$ & P-value \\
\hline \multicolumn{4}{|l|}{ Gender } \\
\hline Male & $20(46.5)$ & $21(56.8)$ & \\
\hline Female & $23(53.5)$ & $16(43.2)$ & \\
\hline \multicolumn{4}{|l|}{ Twin birth } \\
\hline Yes & $1(2.3)$ & $0(0.0)$ & 1.00 \\
\hline \multicolumn{4}{|c|}{ Preeclampsia } \\
\hline Yes & 9 (20.9) & $0(0.0)$ & 0.003 \\
\hline \multicolumn{4}{|c|}{ Decreased fetal movement reported by mother to physician } \\
\hline Yes & $4(9.3)$ & $0(0.0)$ & 0.11 \\
\hline \multicolumn{4}{|c|}{ Abnormal maternal serum triple screen test results } \\
\hline Yes & $6(14.0)$ & $0(0.0)$ & 0.02 \\
\hline \multicolumn{4}{|c|}{ Pre-term delivery ( $<37$ weeks gestation) } \\
\hline Yes & $20(46.5)$ & $0(0.0)$ & $<0.0001$ \\
\hline \multicolumn{4}{|c|}{ Low birth weight $(<2500 \mathrm{~g})$} \\
\hline Yes & $18(41.9)$ & $0(0.0)$ & $<0.0001$ \\
\hline \multicolumn{4}{|c|}{ Small for gestational age (SGA) ( $<$ 10th percentile) } \\
\hline Yes & $11(25.6)$ & $0(0.0)$ & 0.006 \\
\hline \multicolumn{4}{|c|}{ SGA $(<3 r d$ percentile) } \\
\hline Yes & $9(20.9)$ & $0(0.0)$ & 0.003 \\
\hline \multicolumn{4}{|c|}{ Neonatal intensive care unit (NICU) admission } \\
\hline Yes & $11(25.6)$ & $0(0.0)$ & $<0.0001$ \\
\hline
\end{tabular}

aFigures represent actual number of patients while numbers in brackets are percentages.

statistical significance was lost for some tests, probably due to smaller numbers. In this restricted analysis, significantly higher risks were still observed for pre-term delivery $(P=0.002)$, low birth weight $(P<0.0001)$, and NICU admission $(P<0.0001)$ in association with TTD pregnancies, despite small sample sizes (Table 2 ).

Frequencies of gestational complications were also noted among cases with $X P D$-associated combined phenotypes of XP/TTD, XP/CS, and COFS/TTD. Five of six patients with $X P D$-associated XP/TTD were not noted to have any gestational complications; ${ }^{3,8}$ one XPDassociated XP/TTD case was reported as having low birth weight, SGA $<3$ rd percentile, and NICU admission. ${ }^{3}$ Pregnancy with the one patient with COFS/TTD reported in the literature was complicated with preeclampsia, HELLP syndrome, pre-term delivery, low birth weight, SGA $<3$ rd percentile, and NICU admission. ${ }^{8}$ Neonatal history was not reported for the one patient with XP/CS identified through the literature. ${ }^{38}$

\section{Mapping of preeclampsia-associated domains on XPD}

All TTD $(n=8)^{8,20,21}$ and XP $(n=3)^{30,36,37}$ cases with known XPD mutations and explicit information on presence or absence of preeclampsia were included in the analysis to map the preeclampsiaassociated alleles onto XPD. Of the eight TTD (including one COFS/TTD) pregnancies, four were associated with preeclampsia and all eight were associated with at least one prenatal complication. 
Table 2 Comparison of pregnancy complications between TTD and XP cases with mutations in XPD (restricted analysis with 'do not know' answers excluded) ${ }^{\mathrm{a}}$

\begin{tabular}{|c|c|c|c|}
\hline & $\operatorname{TTD}(\mathrm{n}=43)$ & $X P(n=37)$ & P-value \\
\hline \multicolumn{4}{|l|}{ Gender } \\
\hline Male & $20(46.5)$ & $21(58.3)$ & \\
\hline Female & $23(53.5)$ & $15(41.7)$ & \\
\hline \multicolumn{4}{|l|}{ Twin birth } \\
\hline Yes & $1(2.5)$ & $0(0.0)$ & 1.00 \\
\hline No & 39 (97.5) & $7(100.0)$ & \\
\hline \multicolumn{4}{|c|}{ Preeclampsia } \\
\hline Yes & $9(31.0)$ & $0(0.0)$ & 0.30 \\
\hline No & $20(69.0)$ & $6(100.0)$ & \\
\hline \multicolumn{4}{|c|}{ Hemolysis, elevated liver enzymes and low platelets (HELLP) syndrome } \\
\hline No & $23(100.0)$ & $6(100.0)$ & \\
\hline \multicolumn{4}{|c|}{ Decreased fetal movement reported by mother to physician } \\
\hline Yes & $4(44.4)$ & & \\
\hline No & $5(55.6)$ & & \\
\hline
\end{tabular}

\begin{tabular}{|c|c|c|c|}
\hline Yes & $6(60.0)$ & $0(0.0)$ & 0.45 \\
\hline No & $4(40.0)$ & $1(100.0)$ & \\
\hline
\end{tabular}

$\begin{array}{cccc}\text { Pre-term delivery } & (<37 \text { weeks gestation) } & & \\ \text { Yes } & 20(71.4) & 0(0.0) & 0.002 \\ \text { No } & 8(28.6) & 6(100.0) & \end{array}$

Low birth weight $(<2500 \mathrm{~g})$

$\begin{array}{lrll}\text { Yes } & 18(90.0) & 0(0.0) & <0.0001 \\ \text { No } & 2(10.0) & 6(100.0) & \end{array}$

Small for gestational age (SGA) (<10th percentile)

$\begin{array}{lll}\text { Yes } & 11(57.9) & 0(0.0) \\ \text { No } & 8(42.1) & 4(100.0)\end{array}$

SGA (<3rd percentile)

$\begin{array}{lll}\text { Yes } & 9(50.0) & 0(0.0) \\ \text { No } & 9(50.0) & 4(100.0)\end{array}$

Neonatal intensive care unit (NICU) hospitalization Yes $\quad 11(100.0) \quad 0(0.0)$

No $\quad 0(0.0) \quad 4(100.0)$

aFigures represent actual number of patients while numbers in brackets are percentages.

In contrast, none of the three XP pregnancies were associated with preeclampsia or any other gestational complications.

In the four TTD cases with confirmed history of preeclampsia, one of whom also had COFS, the same spatial region of the folded protein was affected; this region is made up of the faces of the helicase domains distal to the DNA-binding sites and includes the amino- and carboxy-terminal portions of the protein (Figure 1a). Biochemical ${ }^{46}$ and biophysical ${ }^{47}$ data have implicated these regions in binding to the CAK complex and the p44 subunit of the core complex of TFIIH.

In two of these TTD cases with preeclampsia, the defect in the protein could be mapped to a region of 20 amino acids in the XPD $\mathrm{C}$-terminus domain (Figure 1a); the genotypes of each of these cases (case 1: L461V, 716-730 $\Delta: A 725 \mathrm{P}$ and case 2: E731R, $\Delta$ ), contained one null allele ${ }^{48}$ and one point mutation each in the C-terminal motif. We searched various databases for homologues of this XPD C-terminus domain, which was associated with preeclampsia. This motif showed strong conservation across species among eukaryotes but was found to be absent from all completed eubacterial genomes in the BLAST database ${ }^{49}$ and in Archaea with the exception of a divergent version of this motif identified in three Methanococcales genomes (Figure 1b). A homologous complex to TFIIH has not been identified in Archaea. ${ }^{50}$

In the two other TTD cases with preeclampsia, the mutations were not adjacent in the primary sequence; however, they mapped along with the C-terminal mutations to the helicase surfaces distal to the DNA-binding sites (Figure 1a). In one case, the genotype consisted of an allele with the $\mathrm{R} 112 \mathrm{H}$ mutation, not associated with preeclampsia (see below), and a T482A, a mutation presumed to be null, on the other allele (Figure 1a). In the COFS/TTD case, a D681W allele was coupled with R616W, another null allele (Figure 1a).

Three of the four TTD cases in our study, which were not associated with preeclampsia were $\mathrm{R} 112 \mathrm{H}$ homozygotes. ${ }^{21}$ In the fourth TTD case, the patient had an allele with severely compromised XPD gene

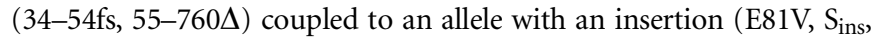
$\mathrm{K} 82 \mathrm{E}$ ) in helix 3 of the second helicase domain. Similar to this latter TTD case, each of the three XP patients had an allele lacking the preeclampsia-associated motif (case 1: G47R:L461V, 716-730 $\Delta$; case 2:

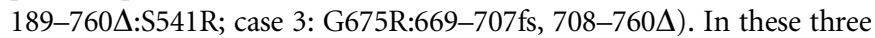
$\mathrm{XP}$ cases, the other alleles all contained point mutations classically associated with XP (case 1: G47R, case 2: S541R, case 3: G675R) (data not shown).

\section{DISCUSSION}

Gestational complications were first reported to be part of the spectrum of abnormalities associated with TTD based on our novel clinical observations and subsequent genetic epidemiologic investigation in TTD families. ${ }^{8}$ In the current study, we found elevated risks of preeclampsia and other placental vascular pregnancy complications in $X P D$-associated TTD- but not XP-affected pregnancies; thus, the exact genetic abnormality is relevant to the underlying mechanism in placental maldevelopment. Our molecular analysis revealed that preeclampsia-associated mutations in XPD affect protein-protein interactions, such as with CAK and p44 subunits of TFIIH, leading to impairment of TFIIH-mediated functions in placenta.

The majority of TTD cases had at least one of the gestational complications noted in Table 1. None of the XPD-associated XP cases were reported as having any of the noted gestational complications. Reporting bias is an unlikely explanation for the observed differences between XP and TTD cases in our study because both conditions are associated with similarly severe (although different) phenotypes.

Genetic susceptibility factors associated with pregnancy complications such as preeclampsia have long been studied with small success, possibly due to the multifactorial causes of the disease. Role of the maternal and fetal genotype in the development of preeclampsia is a topic of heated scientific debate. ${ }^{51-53}$ Here, we demonstrate that at least for TTD-associated mutations in $X P D$, preeclampsia is unequivocally mediated by the fetal genotype. To the best of our knowledge, the penetrance of XPD mutations with respect to preeclampsia is highest compared to mutations reported in other genes.

In effect, the complete absence of pregnancy complications reported in XP patients stands in clear contrast to TTD patients, all of whom had at least one pregnancy complication. These observations also raise important issues concerning the role of the TFIIH complex in normal prenatal development. Different types of mutations in XPD result in the development of either XP or TTD phenotype, with some 
a

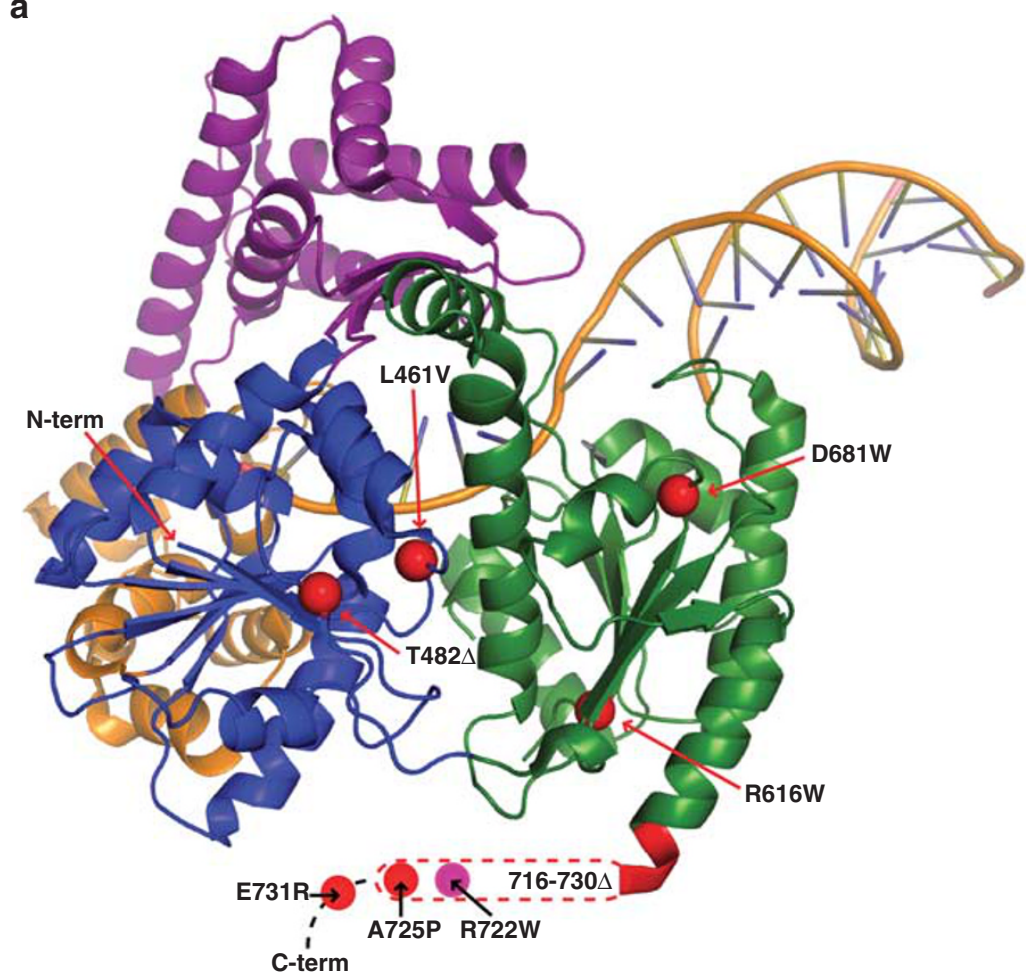

b

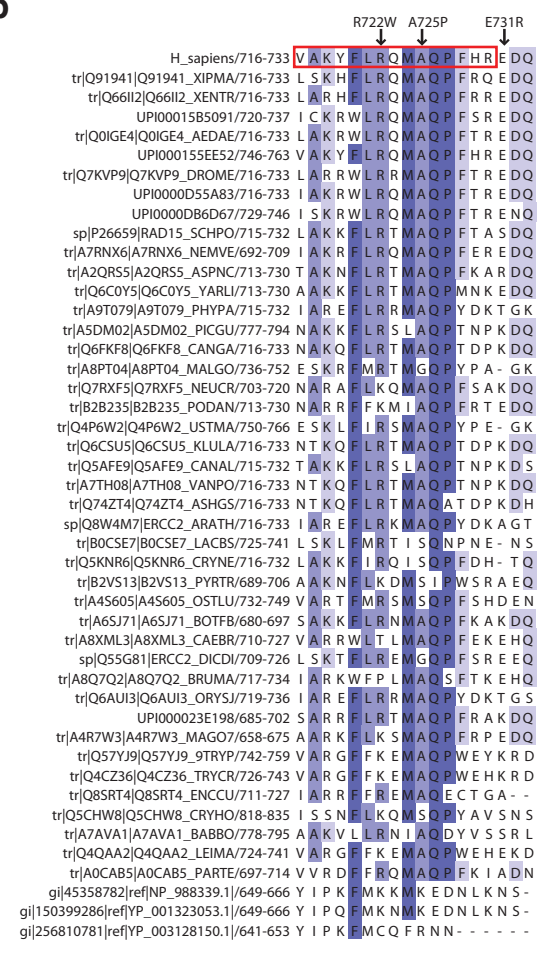

Figure 1 Structural mapping of preeclampsia-associated mutations in XPD. (a) Changes in the amino acid sequences resulting from mutations associated with gestational complications mapped as red spheres to the T. acidophlium XPD crystal structure. ${ }^{43}$ The DNA present in the model was generated by superposition of the T. acidophilum XPD helicase domains with the helicase domains of the co-crystal structure of the Hel308 complex ${ }^{58}$ and is shown to provide general orientation of functional regions. A gross schematic of the region occupied by the C-terminal motif is indicated by red coloring of the amino acids at the T. acidophilum XPD C-terminus and the box outlined in red dashes that follows. The R722W mutation associated with TTD is shown as a pink sphere within the box. The coloring scheme is as follows: helicase 1 domain, blue; $4 \mathrm{Fe}-4 \mathrm{~S}$ domain, gold; arch domain, purple; helicase 2 domain, green; DNA, gold backbone and purple bases. (b) Multiple sequence alignment of the C-terminal motif of eukaryotic XPD proteins. The A725P, E731R mutations associated with gestational complications are indicated with arrows above the alignment. The 716-7304 is outlined in a red box. The R722W mutation associated with TTD maps to this region and is indicated with an arrow above the alignment. The last three sequences in the alignment are putative XPD proteins from Methanococcales species and represent the only prokaryotic XPD-like proteins that could be found with any significant similarity to eukaryotic proteins in this motif. The alignment is colored according to sequence identities and sequences are labeled by their UniRef or GenBank identifiers.

overlapping symptoms. It has been hypothesized that mutations affecting XPD's function in DNA repair cause XP, which is associated with 1000-fold increased risk of skin cancer, while mutations affecting XPD's role in RNA Pol-II-mediated transcription lead to TTD, which is associated with abnormalities of the nervous system, skin and hair. Further experimental studies would be required to address the nature of the cellular dysfunction in placental tissues of TTD patients.

The number of available mutant XPD alleles with confirmed association to preeclampsia was small, but sufficient to allow us to create the first structural map of preeclampsia-associated regions of XPD. Our structural analysis demonstrated a strong association between preeclampsia and a short motif in the C-terminal region of $\mathrm{XPD}$ as well as spatially proximal regions of the helicase domains distal to the DNA-binding sites. These regions are implicated in binding of XPD to the CAK complex and the p44 subunit of the core complex of TFIIH. ${ }^{46,47}$ Individual analysis of each TTD and XP case harboring two mutations in XPD provided further insight into the compensation mechanisms of different XPD alleles. In two TTD cases with preeclampsia, a null allele was coupled with a point mutation in the C-terminal motif. There is some evidence that proteins produced from null mutations retain some function and can complement orthogonal mutations on the sister allele ${ }^{54}$; however, in both of these cases the null alleles lacked a functional complement to the region mutated in the sister alleles. This strongly suggested that the observed preeclampsia in these cases was due to the disruption of a specific function mediated by this C-terminal motif. The absence of this motif in prokaryotes coupled with the experimental data suggests that this motif has a function in eukaryotic transcription.

Disruption of the C-terminal motif alone could not fully account for association with preeclampsia because this motif was not affected in either allele of two of our TTD cases with preeclampsia. However, the regions disrupted in these cases were spatially proximal to the C-terminal motif and were also implicated in transcription by binding of XPD to the CAK complex. Thus, we propose that the alleles associated with preeclampsia in these two cases may be disrupting functions similar to those mediated by the C-terminal motif. In one of those cases, the genotype consisted of a T482 $\Delta$ on one allele and an $\mathrm{R} 112 \mathrm{H}$ mutation on the sister allele. $\mathrm{R} 112 \mathrm{H}$ maps to a residue in contact with the $4 \mathrm{Fe}-4 \mathrm{~S}$ cluster and disrupts the helicase activity of $\mathrm{XPD}^{42}$ Three of the four TTD cases in our study, which were not associated with preeclampsia were $\mathrm{R} 112 \mathrm{H}$ homozygotes. These observations suggested that gestational complications in the R112H:T482A heterozygote TTD case were driven by the T482 $\Delta$ allele. In the COFS/ TTD case, R616W, a null allele, was coupled to D681W on the sister allele; another mutation, D681N, in the same location as D681W has been shown to interfere with p44 binding. ${ }^{55}$ Therefore, the overall result of the mutations in these two cases may be a change in the nature of interaction between XPD and p44; there is evidence that 
failure on the part of XPD to interact with p44 can lead to abolition of transcription without any significant effects on the helicase activity of this protein. ${ }^{56}$

XPD is an evolutionarily conserved ATP-dependent helicase, which is only fully active when assembled in the multi-subunit TFIIH. XPD is specifically stimulated by its interaction with $\mathrm{p} 44^{44,55}$ and its binding to the CAK complex is required for the function of TFIIH in RNA Pol-II-mediated transcription, NER, and phosphorylation of NRs. ${ }^{13,57}$ Future functional studies involving larger sample sizes will determine which particular activities of TFIIH are most severely impacted because of specific mutations in XPD.

Limitations of our study include lack of explicit information on presence or absence of some complications in a subset of subjects; this limitation is unavoidable because gestational complications were not routinely noted during investigation of TTD and XP cases until we reported prenatal complications as part of the spectrum of abnormalities associated with TTD. It would be premature to draw conclusions based on the observation that one of six XP/TTD and the one COFS/TTD case in our study had gestational complications, given the lack of standard diagnostic criteria for and the rarity of these combined phenotypes. Other limitations include a small sample size for structural mapping of preeclampsia-associated domains and the absence of experimental structures for human XPD and XPD/TFIIH/ CAK complexes. Despite these limitations, we obtained consistent and biologically plausible results from our analyses with respect to the involvement of the CAK domain of TFIIH in the development of observed gestational complications.

In summary, our analyses revealed that a specific subset of XPD mutations, which lead to TTD, but are unrelated to XP, result in higher risk of development of preeclampsia and other gestational complications possibly due to their adverse effects on binding of XPD with CAK and p44 subunits of TFIIH, leading to impairment of TFIIH-mediated functions in placenta. Our findings should help drive future research focusing on the role of abnormalities in the various domains of XPD in the development of human prenatal complications.

\section{CONFLICT OF INTEREST}

The authors declare no conflict of interest.

\section{ACKNOWLEDGEMENTS}

This study was supported in part by the Junior Faculty Researcher Award granted to RM through Center for Social and Demographic Analysis (CSDA) at the University at Albany; this award was made possible through a grant (R24 HD044943) from National Institute of Child Health and Human Development at the National Institutes of Health to CSDA.

1 Lehmann AR: DNA repair-deficient diseases, xeroderma pigmentosum, Cockayne syndrome and trichothiodystrophy. Biochimie 2003; 85: 1101-1111.

2 Graham Jr JM, Anyane-Yeboa K, Raams A et al: Cerebro-oculo-facio-skeletal syndrome with a nucleotide excision-repair defect and a mutated XPD gene, with prenatal diagnosis in a triplet pregnancy. Am J Hum Genet 2001; 69: 291-300.

3 Broughton BC, Berneburg M, Fawcett $\mathrm{H}$ et al: Two individuals with features of both xeroderma pigmentosum and trichothiodystrophy highlight the complexity of the clinical outcomes of mutations in the XPD gene. Hum Mol Genet 2001; 10 : 2539-2547.

4 Nouspikel T: Nucleotide excision repair and neurological diseases. DNA Repair (Amst) 2008; 7: 1155-1167.

5 Zurita M, Merino C: The transcriptional complexity of the TFIIH complex. Trends Genet 2003; 19: 578-584.

6 Giglia-Mari G, Coin F, Ranish JA et al: A new, tenth subunit of TFIIH is responsible for the DNA repair syndrome trichothiodystrophy group A. Nat Genet 2004; 36: 714-719.

7 Nikolov DB, Burley SK: RNA polymerase II transcription initiation: a structural view. Proc Natl Acad Sci USA 1997; 94: 15-22.
8 Moslehi R, Signore C, Tamura D et al: Adverse effects of trichothiodystrophy DNA repair and transcription gene disorder on human fetal development. Clin Genet 2010; 77: 365-373.

9 Zhou X, Khan SG, Tamura D et al: Brittle hair, developmental delay, neurologic abnormalities, and photosensitivity in a 4-year-old girl. J Am Acad Dermatol 2010; 63: 323-328.

10 Wakeling EL, Cruwys M, Suri M, Brady AF, Aylett SE, Hall C: Central osteosclerosis with trichothiodystrophy. Pediatr Radiol 2004; 34: 541-546.

11 Fortina AB, Alaibac M, Piaserico S, Peserico A: PIBI(D)S: clinical and molecular characterization of a new case. J Eur Acad Dermatol Venereol 2001; 15: 65-69.

12 Chen E, Cleaver JE, Weber CA et al: Trichothiodystrophy: clinical spectrum, central nervous system imaging, and biochemical characterization of two siblings. J Invest Dermatol 1994; 103: 154S-158S.

13 Botta E, Nardo T, Broughton BC, Marinoni S, Lehmann AR, Stefanini M: Analysis of mutations in the XPD gene in Italian patients with trichothiodystrophy: site of mutation correlates with repair deficiency, but gene dosage appears to determine clinical severity. Am J Hum Genet 1998; 63: 1036-1048.

14 Vermeulen W, Rademakers S, Jaspers NG et al: A temperature-sensitive disorder in basal transcription and DNA repair in humans. Nat Genet 2001; 27: 299-303.

15 Stefanini M, Vermeulen W, Weeda G et al: A new nucleotide-excision-repair gene associated with the disorder trichothiodystrophy. Am J Hum Genet 1993; 53: 817-821.

16 Rebora A, Crovato F: PIBI(D)S syndrome-trichothiodystrophy with xeroderma pigmentosum (group D) mutation. J Am Acad Dermatol 1987; 16: 940-947.

17 Racioppi L, Cancrini C, Romiti ML et al: Defective dendritic cell maturation in a child with nucleotide excision repair deficiency and CD4 Iymphopenia. Clin Exp Immunol 2001; 126: 511-518.

18 Battistella PA, Peserico A: Central nervous system dysmyelination in PIBI(D)S syndrome: a further case. Childs Nerv Syst 1996; 12: 110-113.

19 Tolmie JL, de Berker D, Dawber R et al: Syndromes associated with trichothiodystrophy. Clin Dysmorphol 1994; 3: 1-14.

20 Takayama K, Danks DM, Salazar EP, Cleaver JE, Weber CA: DNA repair characteristics and mutations in the ERCC2 DNA repair and transcription gene in a trichothiodystrophy patient. Hum Mutat 1997; 9: 519-525.

21 Stefanini M, Lagomarsini P, Arlett CF et al: Xeroderma pigmentosum (complementation group D) mutation is present in patients affected by trichothiodystrophy with photosensitivity. Hum Genet 1986; 74: 107-112.

22 Kleijer WJ, Beemer FA, Boom BW: Intermittent hair loss in a child with PIBI(D)S syndrome and trichothiodystrophy with defective DNA repair-xeroderma pigmentosum group D. Am J Med Genet 1994; 52: 227-230.

23 Wagner SD, Elvin JG, Norris P, McGregor JM, Neuberger MS: Somatic hypermutation of Ig genes in patients with xeroderma pigmentosum (XP-D). Int Immunol 1996; 8: 701-705.

24 Pawsey SA, Magnus IA, Ramsay CA, Benson PF, Giannelli F: Clinical, genetic and DNA repair studies on a consecutive series of patients with xeroderma pigmentosum. Q J Med 1979; 48: 179-210.

25 Mamada A, Kondo S, Kawada A, Satoh Y, Fujiwara Y: Delayed sensorineural deafness and skin carcinogenesis in a Japanese xeroderma pigmentosum group $D$ patient. Photodermatology 1988; 5: 83-91.

26 Fukuro S, Yamaguchi J, Mamada A, Kondo S, Satoh Y: Xeroderma pigmentosum group D patient bearing lentigo maligna without neurological symptoms. Dermatologica 1990; 181: 129-133.

27 Emmert S, Ueda T, Zumsteg $U$ et al: Strict sun protection results in minimal skin changes in a patient with xeroderma pigmentosum and a novel c.2009delG mutation in XPD (ERCC2). Exp Dermatol 2009; 18: 64-68.

28 Thielmann HW, Popanda O, Edler L, Jung EG: Clinical symptoms and DNA repair characteristics of xeroderma pigmentosum patients from Germany. Cancer Res 1991; 51: 3456-3470.

29 Nakamura T, Ono T, Yoshimura $\mathrm{K}$ et al: Malignant schwannoma associated with xeroderma pigmentosum in a patient belonging to complementation group $D$. J Am Acad Dermatol 1991; 25: 349-353.

30 Kobayashi T, Kuraoka I, Saijo M et al: Mutations in the XPD gene leading to xeroderma pigmentosum symptoms. Hum Mutat 1997; 9: 322-331.

31 Ishii N, Nakajima H, Kondo S, Satoh Y, Fujiwara Y, Shimoda N: A case of xeroderma pigmentosum group D determined by photobiological study. J Dermatol 1991; 18: 31-35.

32 Ichihashi M, Yamamura K, Hiramoto T, Fujiwara Y: No apparent neurologic defect in a patient with xeroderma pigmentosum complementation group D. Arch Dermatol 1988; 124: 256-260.

33 Fujiwara Y, Satoh Y: Assignment of two Japanese xeroderma pigmentosum patients to complementation group D and their characteristics. Jpn J Cancer Res 1985; 76: 162-166.

34 Fischer E, Thielmann HW, Neundorfer B, Rentsch FJ, Edler L, Jung EG: Xeroderma pigmentosum patients from Germany: clinical symptoms and DNA repair characteristics. Arch Dermatol Res 1982; 274: 229-247.

35 Akiba H, Kato T, Seiji M: Enzyme defects in xeroderma pigmentosum. J Dermatol 1976; 3: 163-170.

36 Fujimoto M, Leech SN, Theron T et al: Two new XPD patients compound heterozygous for the same mutation demonstrate diverse clinical features. J Invest Dermatol 2005; 125: 86-92.

37 Broughton BC, Thompson AF, Harcourt SA et al: Molecular and cellular analysis of the DNA repair defect in a patient in xeroderma pigmentosum complementation group $D$ who has the clinical features of xeroderma pigmentosum and Cockayne syndrome. Am J Hum Genet 1995; 56: 167-174. 
38 Lindenbaum Y, Dickson D, Rosenbaum P, Kraemer K, Robbins I, Rapin I: Xeroderma pigmentosum/cockayne syndrome complex: first neuropathological study and review of eight other cases. Eur J Paediatr Neurol 2001; 5: 225-242.

39 Notredame C, Higgins DG, Heringa J: T-Coffee: a novel method for fast and accurate multiple sequence alignment. J Mol Biol 2000; 302: 205-217.

40 Pei J, Kim BH, Grishin NV: PROMALS3D: a tool for multiple protein sequence and structure alignments. Nucleic Acids Res 2008; 36: 2295-2300.

41 Zhang Y, Skolnick J: TM-align: a protein structure alignment algorithm based on the TM-score. Nucleic Acids Res 2005; 33: 2302-2309.

42 Fan L, Fuss JO, Cheng QJ et al: XPD helicase structures and activities: insights into the cancer and aging phenotypes from XPD mutations. Cell 2008; 133: 789-800.

43 Wolski SC, Kuper J, Hanzelmann P et al: Crystal structure of the FeS cluster-containing nucleotide excision repair helicase XPD. PLoS Biol 2008; 6: e149.

$44 \mathrm{Liu} \mathrm{H}$, Rudolf J, Johnson KA et al: Structure of the DNA repair helicase XPD. Cell 2008; 133: 801-812.

45 Waterhouse AM, Procter JB, Martin DM, Clamp M, Barton GJ: Jalview version 2-a multiple sequence alignment editor and analysis workbench. Bioinformatics 2009; 25: 1189-1191.

46 Sandrock B, Egly JM: A yeast four-hybrid system identifies Cdk-activating kinase as a regulator of the XPD helicase, a subunit of transcription factor IIH. J Biol Chem 2001; 276: 35328-35333.

47 Schultz P, Fribourg S, Poterszman A, Mallouh V, Moras D, Egly JM: Molecular structure of human TFIIH. Cell 2000; 102: 599-607.

48 Taylor EM, Broughton BC, Botta E et al: Xeroderma pigmentosum and trichothiodystrophy are associated with different mutations in the XPD (ERCC2) repair/transcription gene. Proc Natl Acad Sci USA 1997; 94: 8658-8663.
49 Cummings L, Riley L, Black L et al: Genomic BLAST: custom-defined virtual databases for complete and unfinished genomes. FEMS Microbiol Lett 2002; 216 : 133-138.

50 Soppa J: Transcription initiation in Archaea: facts, factors and future aspects. Mol Microbiol 1999; 31: 1295-1305.

51 Oudejans CB, van Dijk M, Oosterkamp M, Lachmeijer A, Blankenstein MA: Genetics of preeclampsia: paradigm shifts. Human Genet 2007; 120: 607-612.

52 Roberts JM, Cooper DW: Pathogenesis and genetics of pre-eclampsia. Lancet 2001; 357: 53-56.

53 Lie RT, Rasmussen S, Brunborg H, Gjessing HK, Lie-Nielsen E, Irgens LM: Fetal and maternal contributions to risk of pre-eclampsia: population based study. BMJ 1998; 316: 1343-1347.

54 Andressoo JO, Jans J, de Wit J et al: Rescue of progeria in trichothiodystrophy by homozygous lethal Xpd alleles. PLoS Biol 2006; 4: e322.

55 Coin F, Marinoni JC, Rodolfo C, Fribourg S, Pedrini AM, Egly JM: Mutations in the XPD helicase gene result in XP and TTD phenotypes, preventing interaction between XPD and the p44 subunit of TFIIH. Nat Genet 1998; 20: 184-188.

56 Dubaele S, Proietti De Santis L, Bienstock RJ et al: Basal transcription defect discriminates between xeroderma pigmentosum and trichothiodystrophy in XPD patients. Mol Cell 2003; 11: 1635-1646.

57 Botta E, Nardo T, Lehmann AR, Egly JM, Pedrini AM, Stefanini M: Reduced level of the repair/transcription factor TFIIH in trichothiodystrophy. Hum Mol Genet 2002; 11: 2919-2928.

58 Buttner K, Nehring S, Hopfner KP: Structural basis for DNA duplex separation by a superfamily-2 helicase. Nat Struct Mol Biol 2007; 14: 647-652. 